\title{
Leptonic mono-top from single stop production at the LHC
}

\author{
Guang Hua Duan, ${ }^{c}$ Ken-ichi Hikasa, ${ }^{d}$ Lei Wu, ${ }^{a, b}$ Jin Min Yang $^{c, d}$ and \\ Mengchao Zhang ${ }^{e}$ \\ ${ }^{a}$ Department of Physics and Institute of Theoretical Physics, Nanjing Normal University, \\ Nanjing, Jiangsu 210023, China \\ ${ }^{b} A R C$ Centre of Excellence for Particle Physics at the Terascale, \\ School of Physics, The University of Sydney, \\ NSW 2006, Australia \\ ${ }^{c}$ CAS Key Laboratory of Theoretical Physics, Institute of Theoretical Physics, \\ Chinese Academy of Sciences, \\ Beijing 100190, China \\ ${ }^{d}$ Department of Physics, Tohoku University, \\ Sendai 980-8578, Japan \\ ${ }^{e}$ Center for Theoretical Physics and Universe, Institute for Basic Science (IBS), \\ Daejeon 34051, Korea \\ E-mail: ghduan@itp.ac.cn, hikasa@phys.tohoku.ac.jp, \\ lei.wu1@sydney.edu.au, jmyang@itp.ac.cn, mczhang@ibs.re.kr
}

ABSTRACT: Top squark (stop) can be produced via QCD interaction but also the electroweak interaction at the LHC. In this paper, we investigate the observability of the associated production of stop and chargino, $p p \rightarrow \tilde{t}_{1} \tilde{\chi}_{1}^{-}$, in compressed electroweakino scenarios at $14 \mathrm{TeV}$ LHC. Due to small mass splitting between the lightest neutralino $\left(\tilde{\chi}_{1}^{0}\right)$ and chargino $\left(\tilde{\chi}_{1}^{-}\right)$, the single stop production can give the mono-top signature through the stop decay $\tilde{t}_{1} \rightarrow t \tilde{\chi}_{1}^{0}$. We analyze the leptonic mono-top channel of the single stop production and propose a lab-frame observable $\cos \theta_{b \ell}$ to reduce the SM backgrounds. We find that such leptonic mono-top events from the single stop production can be probed at $2 \sigma$ level at the HL-LHC if $m_{\tilde{t}_{1}}<760 \mathrm{GeV}$ and $m_{\tilde{\chi}_{1}^{0}}<150 \mathrm{GeV}$. Given a discovery of the stop and a measurement of the single stop production cross section, the stop mixing angle can also be determined from the single stop production at the HL-LHC.

KeYWORDs: Supersymmetry Phenomenology

ArXiv EPrint: 1611.05211 


\section{Contents}

2 Single production and decays of stop in compressed electroweakino scenario

3 Leptonic mono-top signature from single stop production at the LHC

\section{Introduction}

After the discovery of the Higgs boson in 2012 [1, 2], the fundamental mechanism of stabilizing the electroweak scale has become an urgent topic. Weak scale supersymmetry (SUSY) is one of the most promising candidates for addressing such an longstanding theoretical issue. SUSY predicts a plethora of supersymmetric particles, among which the top-squarks (stops) play an important role in cancelling the quadratic divergence in the Higgs boson mass. Naturalness (absence of fine-tuning in the Higgs boson mass) requires stop masses to be below $1 \mathrm{TeV}$ in the MSSM [3]. Therefore, the search for light stops is a sensitive probe of the naturalness in SUSY [4-23].

So far, ATLAS and CMS collaborations have performed extensive searches for stops at the LHC Run-1 and Run-2. The current search strategies are specialized for different kinematical regions. For example, when $m_{\tilde{t}_{1}} \gg m_{\tilde{\chi}_{1}^{0}}+m_{t}$, the top quarks from stop decays are usually energetic. With the endpoint observables, such as $M_{T 2}$ [24, 25], the stop pair can be discriminated from the $t \bar{t}$ background. But in the compressed regions, for example $m_{\tilde{t}_{1}} \approx m_{\tilde{\chi}_{1}^{0}}+m_{t}$, the decay products of the stop are very soft. In this region, the stop is searched for by using the monojet signature [26-32]. Based on recent Run-2 $\left(\sim 15 \mathrm{fb}^{-1}\right)$ dataset, the stop mass has been excluded up to $\sim 1 \mathrm{TeV}$ in simplified models [33-40].

Like the top quark, stops can be produced in pair, but also can be singly produced via the electroweak interaction, such as the associated production of the stop and chargino, $b g \rightarrow \tilde{t}_{1} \tilde{\chi}_{1}^{-}$(cf. figure 1) [41-43]. When the stop and chargino are not heavy or the chargino is much lighter than the stop [41], the single stop production can have a sizable cross section at the LHC. Although the single stop may not be a good discovery channel as the stop pair production, the study of single stop is meaningful because it can serve as a complementary channel to probe the electroweak properties of the stop [42, 43].

In this work, we explore the feasibility of probing the single stop production process $p p \rightarrow \tilde{t}_{1}\left(\rightarrow t \tilde{\chi}_{1}^{0}\right) \tilde{\chi}_{1}^{-}+X$ in a compressed SUSY scenario, where the chargino $\tilde{\chi}_{1}^{ \pm}$is almost degenerate with the lightest neutralino $\tilde{\chi}_{1}^{0}$. Such a spectrum is motivated by natural SUSY $[4,5]$ or the well-tempered neutralino frameworks [44]. Due to the small mass splitting between $\tilde{\chi}_{1}^{ \pm}$and $\tilde{\chi}_{1}^{0}$, the single stop production will give the mono-top signature [43, 45-49] and in ref. [43] its full-hadronic final states with top tagging technique are 


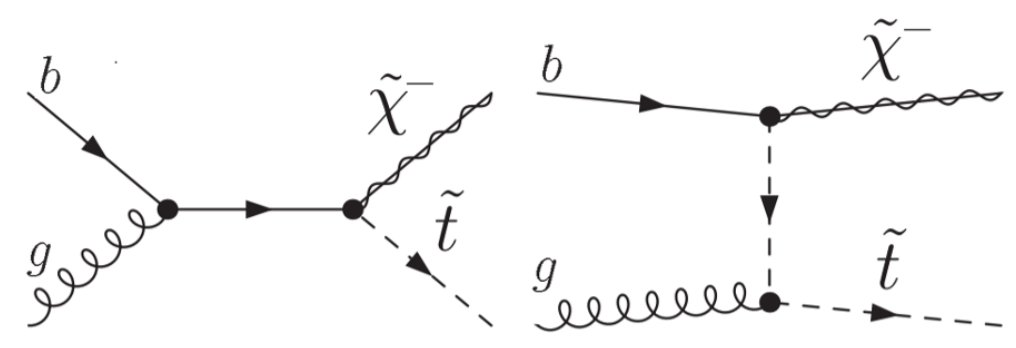

Figure 1. Feynman diagrams for the associated production of the stop and chargino at the LHC.

studied. In this study, we focus on the leptonic mono-top channel of the process $p p \rightarrow \tilde{t}_{1} \tilde{\chi}_{1}^{-}$. In contrast with the full hadronic channel, the leptonic channel has no QCD background pollution. Besides, the cut on the leptonic $m_{T}$ can greatly reduce the $t \bar{t}$ and $W+b$ backgrounds [47, 48]. We also construct a new variable, which is the open angle of the charged lepton and $b$-jet from the top quark in the stop decay, to reduce the SM backgrounds.

This work is organized as follows. In section 2, we calculate the single stop production at the LHC and stop decays in compressed electroweakino scenarios. In section 3, we perform detailed Monte Carlo simulation for the leptonic mono-top signature from the single stop production at the LHC. Finally, we summarize our conclusions in section 4 .

\section{Single production and decays of stop in compressed electroweakino scenario}

In the MSSM, the stop mass matrix in the gauge-eigenstate basis $\left(\tilde{t}_{L}, \tilde{t}_{R}\right)$ is given by

$$
M_{\tilde{t}}^{2}=\left(\begin{array}{cc}
m_{\tilde{t}_{L}}^{2} & m_{t} X_{t}^{\dagger} \\
m_{t} X_{t} & m_{\tilde{t}_{R}}^{2}
\end{array}\right)
$$

with

$$
\begin{aligned}
m_{\tilde{t}_{L}}^{2} & =m_{\tilde{Q}_{3 L}}^{2}+m_{t}^{2}+m_{Z}^{2}\left(\frac{1}{2}-\frac{2}{3} \sin ^{2} \theta_{W}\right) \cos 2 \beta, \\
m_{\tilde{t}_{R}}^{2} & =m_{\tilde{U}_{3 R}}^{2}+m_{t}^{2}+\frac{2}{3} m_{Z}^{2} \sin ^{2} \theta_{W} \cos 2 \beta, \\
X_{t} & =A_{t}-\mu \cot \beta .
\end{aligned}
$$

Here $m_{\tilde{Q}_{3 L}}$ and $m_{\tilde{U}_{3 R}}$ are the soft-breaking mass parameters for the third generation lefthanded squark doublet $\tilde{Q}_{3 L}$ and the right-handed stop $\tilde{U}_{3 R}$, respectively. $A_{t}$ is the stop soft-breaking trilinear parameter. The generation mixing is neglected here. This hermitian matrix can be diagonalized by a unitary transformation:

$$
\left(\begin{array}{c}
\tilde{t}_{1} \\
\tilde{t}_{2}
\end{array}\right)=\left(\begin{array}{cc}
\cos \theta_{\tilde{t}} & \sin \theta_{\tilde{t}} \\
-\sin \theta_{\tilde{t}} & \cos \theta_{\tilde{t}}
\end{array}\right)\left(\begin{array}{c}
\tilde{t}_{L} \\
\tilde{t}_{R}
\end{array}\right),
$$

where $\theta_{\tilde{t}}$ is the mixing angle between left-handed $\left(\tilde{t}_{L}\right)$ and right-handed $\left(\tilde{t}_{R}\right)$ stops. In the mass eigenstates, the relevant interactions of the stop and electroweakinos are given by

$$
\begin{aligned}
\mathcal{L}_{\tilde{t}_{1} \bar{b} \tilde{\chi}_{i}^{+}} & =\tilde{t}_{1} \bar{b}\left(f_{L}^{C} P_{L}+f_{R}^{C} P_{R}\right) \tilde{\chi}_{i}^{+}+\text {h.c. }, \\
\mathcal{L}_{\tilde{t}_{1} \bar{t} \tilde{\chi}_{i}^{0}} & =\tilde{t}_{1} \bar{t}\left(f_{L}^{N} P_{L}+f_{R}^{N} P_{R}\right) \tilde{\chi}_{i}^{0}+\text { h.c. },
\end{aligned}
$$


where $P_{L / R}=\left(1 \mp \gamma_{5}\right) / 2$, and

$$
\begin{aligned}
f_{L}^{N} & =-\left[\frac{g_{2}}{\sqrt{2}} N_{i 2}+\frac{g_{1}}{3 \sqrt{2}} N_{i 1}\right] \cos \theta_{\tilde{t}}-y_{t} N_{i 4} \sin \theta_{\tilde{t}} \\
f_{R}^{N} & =\frac{2 \sqrt{2}}{3} g_{1} N_{i 1}^{*} \sin \theta_{\tilde{t}}-y_{t} N_{i 4}^{*} \cos \theta_{\tilde{t}}, \\
f_{L}^{C} & =y_{b} U_{i 2}^{*} \cos \theta_{\tilde{t}}, \\
f_{R}^{C} & =-g_{2} V_{i 1} \cos \theta_{\tilde{t}}+y_{t} V_{i 2} \sin \theta_{\tilde{t}},
\end{aligned}
$$

with $y_{t}=\sqrt{2} m_{t} /(v \sin \beta)$ and $y_{b}=\sqrt{2} m_{b} /(v \cos \beta)$ being the top and bottom Yukawa couplings, respectively. When $\tan \beta$ is large, the values of $y_{b}$ can be sizable. The neutralino and chargino mixing matrices $N_{i j}, U_{i j}, V_{i j}$ are defined in [50]. The compressed electroweakino spectrum, $m_{\tilde{\chi}_{1}^{ \pm}}-m_{\tilde{\chi}_{1}^{0}} \ll m_{\tilde{\chi}_{1}^{0}}$, can be realized in two limits:

(i) $\mu \ll M_{1,2}, V_{11}, U_{11}, N_{11,12,21,22} \sim 0, V_{12} \sim \operatorname{sgn}(\mu), U_{12} \sim 1$ and $N_{13,14,23}=-N_{24} \sim$ $1 / \sqrt{2}$. In this limit, the two neutralinos $\tilde{\chi}_{1,2}^{0}$ and the chargino $\tilde{\chi}_{1}^{ \pm}$are nearly degenerate higgsinos $\left(\tilde{H}^{ \pm}\right)$. Such a higgsino LSP scenario may be probed at the high luminosity LHC [51-55].

(ii) $M_{2} \ll \mu, M_{1}, V_{11}, U_{11} \sim 1, V_{12}, U_{12} \sim 0, N_{11,13,14}, N_{22,23,24} \sim 0$, and $N_{12,21} \sim 1$. In this case, the lightest neutralino $\tilde{\chi}_{1}^{0}$ and the lighter chargino $\tilde{\chi}_{1}^{ \pm}$are nearly degenerate winos $\left(\tilde{W}^{ \pm}\right)$. If the small splitting between $\tilde{\chi}_{1}^{ \pm}$and $\tilde{\chi}_{1}^{0}$ is not too small, the monojet with soft photon events can be used to detect this wino LSP scenario at the LHC [56-58].

We evaluate the mass spectrum and branching ratios of all sparticles with SUSYHIT [60]. We use MadGraph5_aMCONLO [61] to calculate the leading order cross section of the single stop production. The NNPDF23LO1 [62] parton distribution functions are chosen for our calculations. The renormalization and factorization scales are set as the default value. We include the NLO-QCD correction by applying a $K$-factor of 1.3 [41, 43] to the cross section of the single stop production. It should be noted that the single stop production not only relies on the nature of the electroweakinos, but also is affected by the polarized states of the stop. To demonstrate this, we consider two cases: the lefthanded stop $\tilde{t}_{L}$ by taking $m_{\tilde{U}_{3 R}}=2 \mathrm{TeV}$ to decouple the right-handed component, and the right-handed stop $\tilde{t}_{R}$ by taking $m_{\tilde{Q}_{3 L}}=2 \mathrm{TeV}$ to decouple the left-handed component.

In the upper panels of figure 2 , we show the cross sections of the associated production of stop and chargino at $14 \mathrm{TeV}$ LHC for four different final states: $\tilde{t}_{L} \tilde{H}^{-}, \tilde{t}_{R} \tilde{H}^{-}, \tilde{t}_{L} \tilde{W}^{-}$ and $\tilde{t}_{R} \tilde{W}^{-}$. The contributions of the conjugate processes are included. For a higgsino-like chargino, we can see that the cross section of $\tilde{t}_{R} \tilde{H}^{-}$production is larger than that of $\tilde{t}_{L} \tilde{H}^{-}$ production and almost independent of $\tan \beta$. It can reach about $3 \mathrm{pb}$ at $m_{\tilde{t}_{1}}=200 \mathrm{GeV}$. While the cross section of $\tilde{t}_{L} \tilde{H}^{-}$strongly depends on the value of $\tan \beta$, since the coupling of the left-handed stop with $\tilde{\chi}_{1}^{ \pm}$is dominated by the bottom Yukawa coupling $y_{b}$ and can be enhanced by a large $\tan \beta$. For a wino-like chargino, the cross section of $\tilde{t}_{L} \tilde{W}^{-}$is always much larger than that of $\tilde{t}_{R} \tilde{W}^{-}$because of the gauge interactions. 

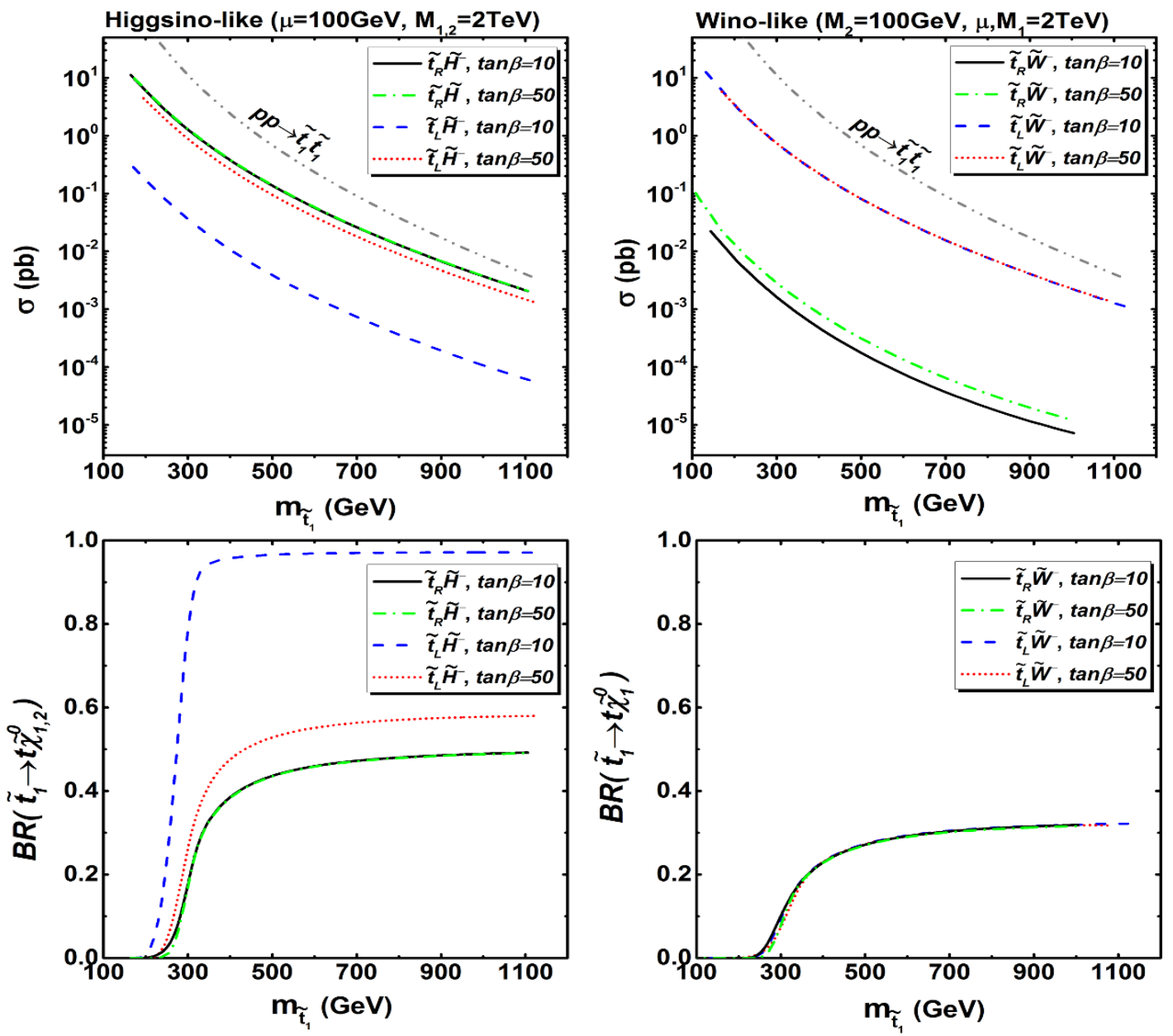

Figure 2. Cross sections of the associated production of stop and chargino at $14 \mathrm{TeV}$ LHC (upper panel), and the stop decay branching ratios (lower panel) in two compressed electroweakino scenarios, where $\tan \beta=10$ and 50. The left (right) two figures are for a higgsino-like (wino-like) chargino $\tilde{\chi}_{1}^{ \pm}$.

In the lower panel of figure 5, we present the branching ratios of stop decaying to the top quark and neutralinos. For higgsino case, it can be seen that a left-handed stop $\tilde{t}_{L}$ dominantly decays to $t \tilde{\chi}_{1,2}^{0}$ at $\tan \beta=10$. The reason is that the decay width of $b \tilde{\chi}_{1}^{+}$is proportional to $y_{b}$ and is suppressed for a small $\tan \beta$. If the stop is right-handed $\tilde{t}_{R}$, its couplings with $\tilde{\chi}_{1,2}^{0}$ and $\tilde{\chi}_{1}^{ \pm}$are proportional to $y_{t}$, and the branching ratios of $\tilde{t}_{R} \rightarrow t \tilde{\chi}_{1,2}^{0}$ and $\tilde{t}_{R} \rightarrow b \tilde{\chi}_{1}^{+}$are about $50 \%$ and $50 \%$, respectively. For the wino case, both $\tilde{t}_{L}$ and $\tilde{t}_{R}$ decay to $t \tilde{\chi}_{1}^{0}$ with the same branching ratio.

Besides, it can be seen that the cross section of stop pair production $\sigma\left(\tilde{t}_{t^{*}}\right)$ is about one order of magnitude larger than that of single stop production if stop mass is less than $300 \mathrm{GeV}$. With the increase of stop mass, the cross section of stop pair production decreases more rapidly than the single stop production due to the suppression of phase space. For example, when stop mass is $700 \mathrm{GeV}$, the ratio of $\sigma\left(\tilde{t} \tilde{t}^{*}\right) / \sigma\left(\tilde{t}_{R} \tilde{H}^{-}\right)$is about four. Considering the stop decay branching ratios, we find that the number of events of $\tilde{t}_{1} \tilde{t}_{1}^{*}\left(\rightarrow \bar{t} \tilde{\chi}_{1,2}^{0}\right)$ 
production is still about two times larger than that of $\tilde{t}_{R}\left(\rightarrow t \tilde{\chi}_{1,2}^{0}\right) \tilde{H}^{-}$production. While the expected number of events of $\tilde{t}_{L}\left(\rightarrow t \tilde{\chi}_{1,2}^{0}\right) \tilde{H}^{-}$and $\tilde{t}_{L, R}\left(\rightarrow t \tilde{\chi}_{1}^{0}\right) \tilde{W}^{-}$productions are less than that of $\tilde{t}_{R}\left(\rightarrow t \tilde{\chi}_{1,2}^{0}\right) \tilde{H}^{-}$. In the following, we will use $\tilde{t}_{R}\left(\rightarrow t \tilde{\chi}_{1,2}^{0}\right) \tilde{H}^{-}$production as an example to investigate the observability of the single stop production at the LHC.

\section{Leptonic mono-top signature from single stop production at the LHC}

Since $\tilde{\chi}_{1}^{ \pm}$and $\tilde{\chi}_{1,2}^{0}$ are the nearly degenerate higgsinos in our considered scenario, the mass splitting between them is small so that $\tilde{\chi}_{1}^{ \pm}$and $\tilde{\chi}_{2}^{0}$ appear as missing transverse energy at the LHC. This leads to the mono-top signature for the single stop production at the LHC, which is

$$
p p \rightarrow \tilde{t}_{1}\left(\rightarrow t \tilde{\chi}_{1,2}^{0}\right) \tilde{\chi}_{1}^{-} \rightarrow t+\mathbb{E}_{T}
$$

In our simulation, we focus on the leptonic mono-top channel. In contrast with the full hadronic final states, the problematic QCD multijet background can be safely neglected in this leptonic channel. We use MadGraph5_aMC@NLO [61] to generate the parton level events. Then, we perform the parton shower and hadronization by Pythia [63]. The jets are clustered by the anti- $k_{t}$ algorithm with a cone radius $\Delta R=0.4$ [65]. We implement the detector effects with Delphes [64].

The SM backgrounds are dominated by the following processes:

- The largest SM backgrounds are the semi- and di-leptonic $t \bar{t}$ productions, where the missing lepton and the limited jet energy resolution will lead to relatively large missing $E_{T}$. The leading order cross section of $t \bar{t}$ production is normalized to its approximate next-to-next-to-leading-order value $\sigma_{t \bar{t}}^{\text {NNLOapprox }}=920 \mathrm{pb}[66]$.

- The subdominant background is the single top production, which is irreducible, up to a jet that could come from ISR. We include three production modes $t j, t b$ and $t W$ in our simulation.

There are other possible SM backgrounds, such as $W+$ jets and the diboson production $V V$. But for $W+$ jets, the mistag rate of a light jet as a $b$-jet in current ATLAS and CMS analyses is of the order of $10^{-2}$ and $10^{-3}$, depending on the working point of the $b$-tagging algorithm. The acceptance of this background after cuts is found to be negligibly small. On the other hand, $V V$ backgrounds can also be neglected because of their small cross sections and the difficulty of faking a $b \ell \mathbb{E}_{T}$ final state in $W W, W Z$ and $Z Z$ backgrounds.

In figure 3 , we present the distributions of the transverse missing energy $\mathbb{E}_{T}$ and the transverse mass of the lepton plus missing energy system $M_{T}^{\ell}$. It is clear that the backgrounds and the signal can be discriminated by $\mathbb{E}_{T}$. Most events of the backgrounds are distributed in the region of $\mathbb{E}_{T} \lesssim 150 \mathrm{GeV}$. However, the signal has much more events than backgrounds in the region of $\mathbb{E}_{T} \gtrsim 150 \mathrm{GeV}$, due to the extra missing energy from the massive LSP. Besides, the variable $M_{T}^{\ell}$ can well separate the backgrounds and signal because it has an end-point at the mass of the lepton's parent particle, $\left.M_{T}^{\ell}\right|_{\max }=M[67]$. All the main backgrounds contain a $W$ boson and a unique source of missing energy, the neutrino, coming from its decay. So the backgrounds have endpoint around $M_{W}$ in the 

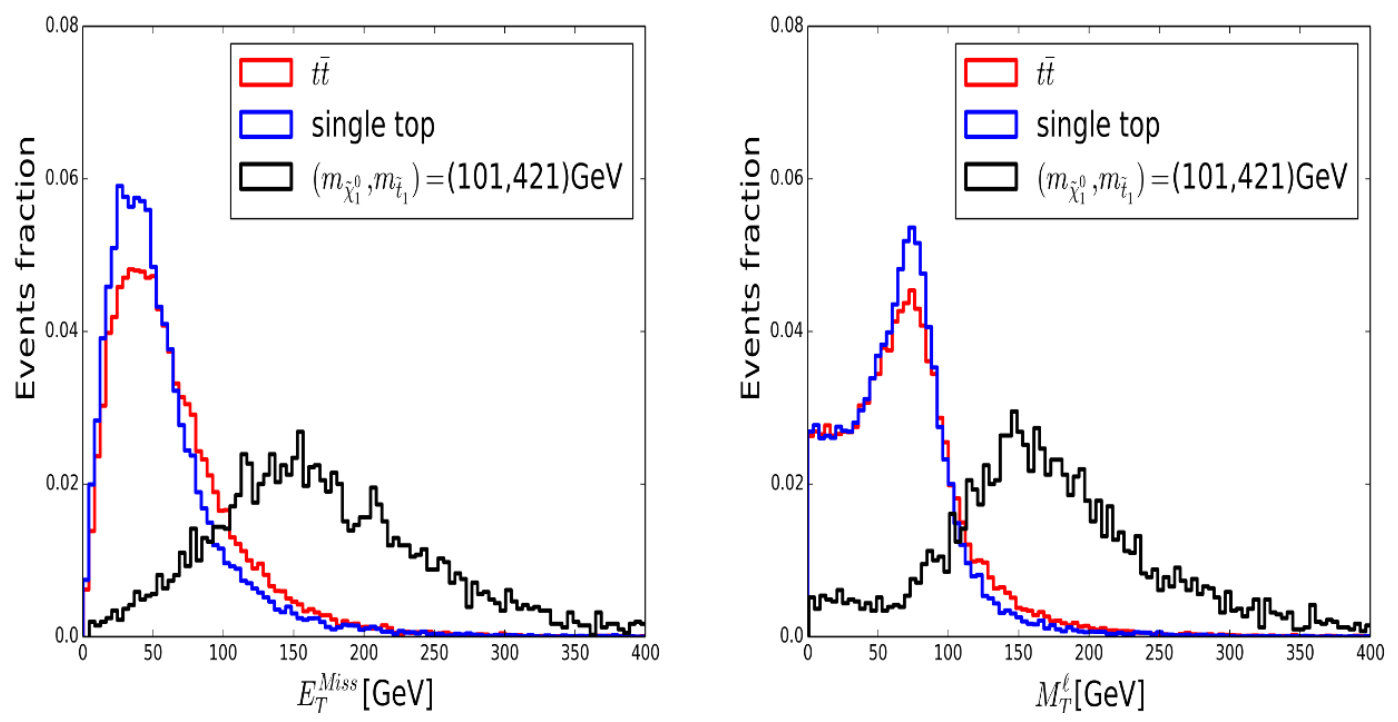

Figure 3. Distributions of transverse missing energy $\mathbb{E}_{T}$ and the transverse mass of the lepton plus missing energy system $M_{T}^{\ell}$. The signal benchmark point is for $m_{\tilde{\chi}_{1}^{0}}=101 \mathrm{GeV}$ and $m_{\widetilde{t}_{1}}=421 \mathrm{GeV}$.

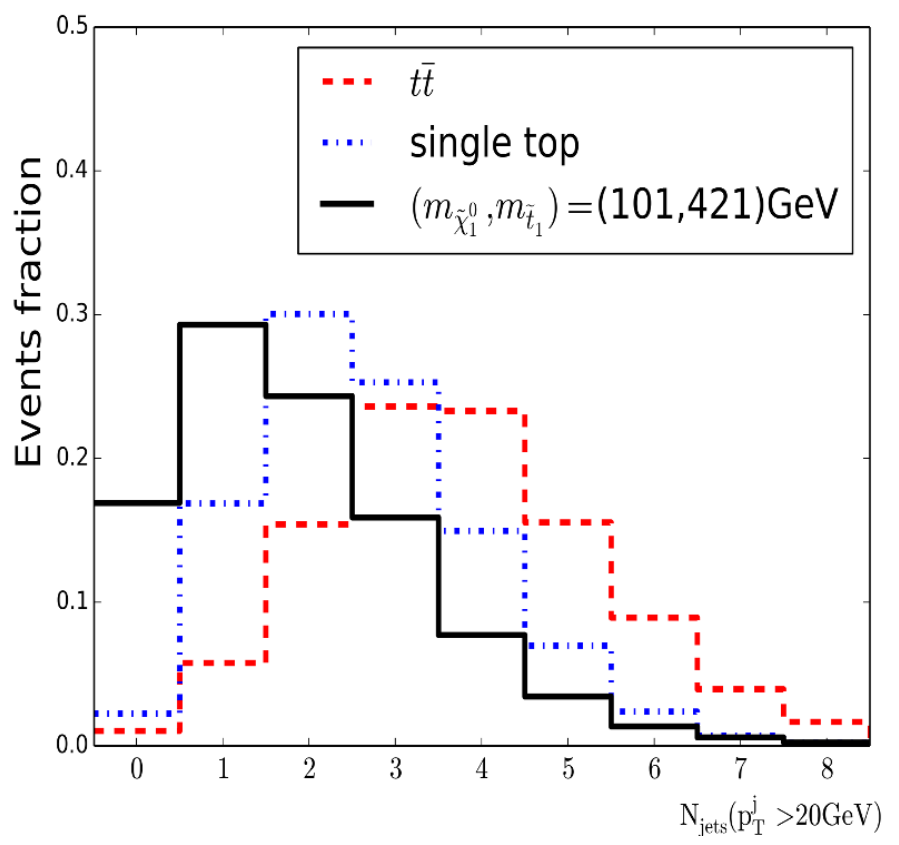

Figure 4. Same as figure 3, but for the distribution of jet multiplicity.

$M_{T}^{\ell}$ distributions. But the signal has a larger value of $M_{T}^{\ell}$. A cut on $M_{T}^{\ell} \geq 80 \mathrm{GeV}$ will greatly reduce the backgrounds while keep most of the signal.

In figure 4 , we show the jet multiplicity $\left(N_{\text {jets }}\right)$ distributions of the signal and backgrounds. We can see that most of events of $t \bar{t}$ and single top backgrounds have larger $N_{\text {jets }}$ than the single stop process. To suppress the backgrounds, we will veto the second hard jet in our event selection. 


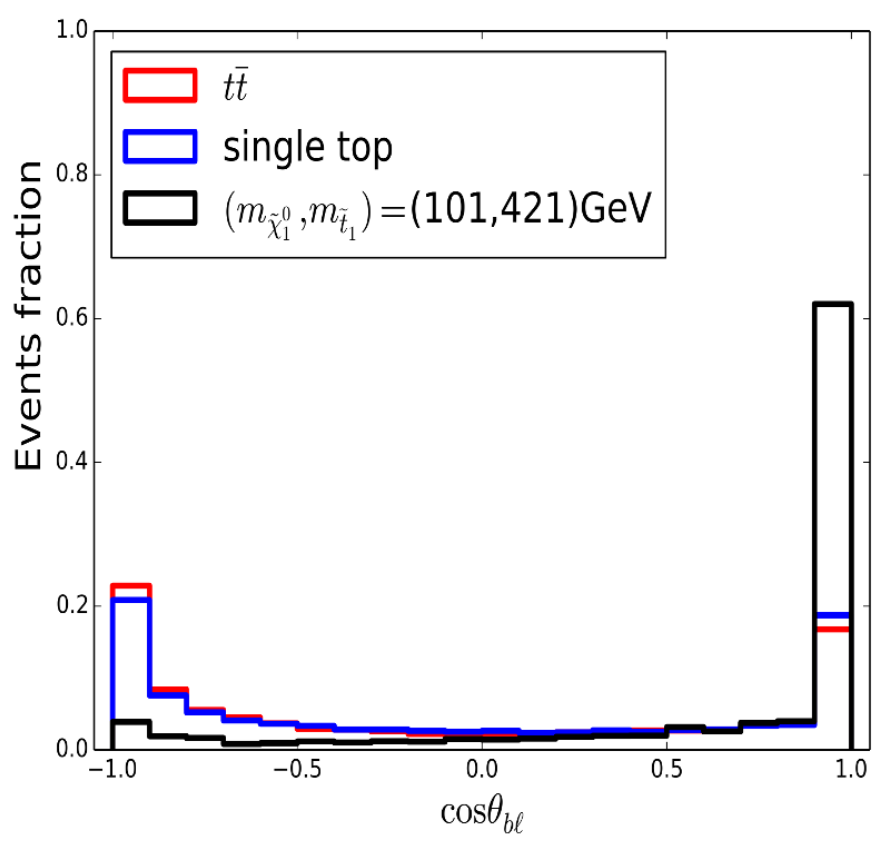

Figure 5. Same as figure 3, but for the distribution of the opening angle $\cos \theta_{b \ell}$ between the charged lepton and the $b$-jet in the lab-frame.

Another interesting observable is the opening angle $\theta_{b \ell}$ between the charged lepton and the $b$-jet in the lab-frame. After requiring exactly one lepton and one $b$-jet, we display the distribution of $\cos \theta_{b \ell}$ in figure 5 . We can see that most of the signal events fall in the region of $\cos \theta_{b \ell}>0$, while the backgrounds have more events in the region of $\cos \theta_{b \ell}<0$. This is because the the charged lepton and the $b$-quark from top quark in the stop decays are boosted so that they tend to move in the same direction when the mass splitting between $\tilde{t}_{1}$ and $\tilde{\chi}_{1}^{0}$ is large. Thus, the requirement of a large $\cos \theta_{b \ell}$ can further reduce backgrounds.

The detailed analysis strategies are the followings:

- We require exact one hard lepton with $p_{T}(\ell)>30 \mathrm{GeV}$ and $\left|\eta_{\ell}\right|<2.5$.

- We require exact one $b$-jet with $p_{T}(b)>75 \mathrm{GeV}$ and $\left|\eta_{b}\right|<2.5$ and veto extra jets with $p_{T}(j)>20 \mathrm{GeV}$ to suppress the $t \bar{t}$ background.

- We define eight signal regions according to $\mathbb{E}_{T}$ cuts: $150,175,195,200,205,225,250$ and $275 \mathrm{GeV}$. It is worth noting at this point that cuts in $M_{T}$ end up having little correlation with cuts in $\mathbb{E}_{T}$, as it will be shown in the cut-flow tables below.

- We require $M_{T}^{\ell}>175 \mathrm{GeV}$ and $\cos \theta_{b \ell}>0.85$ to suppress top pair and single stop backgrounds.

Finally, we use the signal region with highest $S / \sqrt{B}$ to show our results in figure 6 .

In table 1, we present a cut flow of cross sections for the signal and backgrounds at the $14 \mathrm{TeV}$ LHC. The benchmark point is $m_{\tilde{\chi}_{1}^{0}}=101 \mathrm{GeV}$ and $m_{\widetilde{t}_{1}}=421 \mathrm{GeV}$. We can see that the $t \bar{t}$ production is the largest SM background. The requirement of exact one $b$-jet with 


\begin{tabular}{|c|c|c|c|c|c|c|}
\hline cut & $\begin{array}{c}1 \text { lepton } \\
p_{T}^{\ell}>30 G e V,\left|\eta^{\ell}\right|<2.5\end{array}$ & $\begin{array}{c}1 b \text {-jet } \\
p_{T}^{b}>75 G e V,\left|\eta^{b}\right|<2.5\end{array}$ & $\begin{array}{c}\text { jet veto } \\
p_{T}(j)>20 \mathrm{GeV}\end{array}$ & $\begin{array}{c}M_{T}^{\ell}>175 \\
{[\mathrm{GeV}]}\end{array}$ & $\begin{array}{c}\mathbb{E}_{T}>150 \\
{[\mathrm{GeV}]}\end{array}$ & $\begin{array}{c}\cos \theta_{b \ell} \\
>0.85\end{array}$ \\
\hline$t \bar{t}$ & 233465.16 & 77973.38 & 796.20 & 62.95 & 26.2472 & 11.75 \\
\hline$t+j / b / W$ & 44891.80 & 8411.10 & 189.24 & 9.45 & 3.47 & 1.64 \\
\hline signal & 24.88 & 9.482 & 1.40 & 1.03 & 0.90 & 0.77 \\
\hline $\mathrm{S} / \mathrm{B}(\%)$ & & & & 1.43 & 3.02 & 5.75 \\
$S / \sqrt{B}$ & & & & 6.67 & 9.04 & 11.53 \\
\hline
\end{tabular}

Table 1. A cut flow analysis of the cross sections of the backgrounds and signal at $14 \mathrm{TeV}$ LHC, where the cross sections are in unit of $\mathrm{fb}$. The significance $S / \sqrt{B}$ is calculated assuming $3000 \mathrm{fb}^{-1}$ of integrated luminosity. The benchmark point is $\left(m_{\tilde{\chi}_{1}^{0}}, m_{\tilde{t}_{1}}\right)=(101,421) \mathrm{GeV}$.

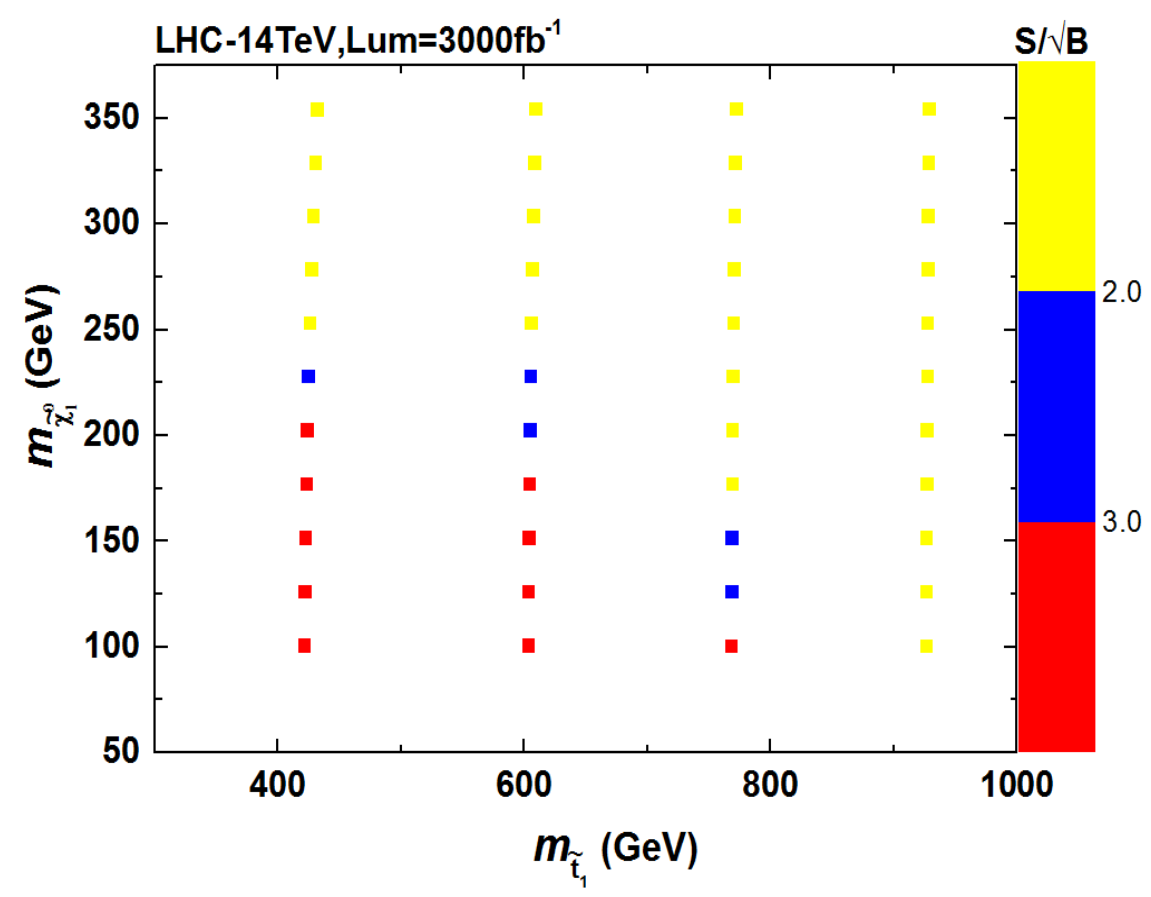

Figure 6. The statistical significance $S / \sqrt{B}$ on the plane of $m_{\tilde{t}_{1}}$ versus $m_{\tilde{\chi}_{1}^{0}}$ at $14 \mathrm{TeV} \mathrm{LHC}$ with $\mathcal{L}=3000 \mathrm{fb}^{-1}$.

$p_{T}^{b}>75 \mathrm{GeV}$ can reduce the backgrounds by about $60 \%$. The jet-veto for the second hard jet can significantly reduce $t \bar{t}$ background by almost two orders of magnitude. The cuts of $M_{T}^{\ell}>175 \mathrm{GeV}$ and $\mathbb{E}_{T}>150 \mathrm{GeV}$ can further remove the backgrounds by one order of magnitude. It should be noted that $\cos \theta_{b \ell}>0.85$ can help to suppress backgrounds by half and improve the value of $S / B$.

In figure 6 , we plot the dependence of the signal significance $S / \sqrt{B}$ on $m_{\tilde{\chi}_{1}^{0}}$ and $m_{\widetilde{t}_{1}}$ for the $14 \mathrm{TeV}$ LHC with a luminosity $\mathcal{L}=3000 \mathrm{fb}^{-1}$. From this figure we can see that the significance drops with the increase of $m_{\tilde{\chi}_{1}^{0}}$ and $m_{\widetilde{t}_{1}}$ because of the reduction of the cross section. We find that the parameter range $100 \mathrm{GeV} \leq m_{\tilde{\chi}_{1}^{0}} \leq 150 \mathrm{GeV}$ and $m_{\tilde{t}_{1}} \leq 760 \mathrm{GeV}$ can be covered at $\geq 2 \sigma$ level with $S / B>3 \%$ at the HL-LHC, which is moderately better than the hadronic stop channel [43]. 


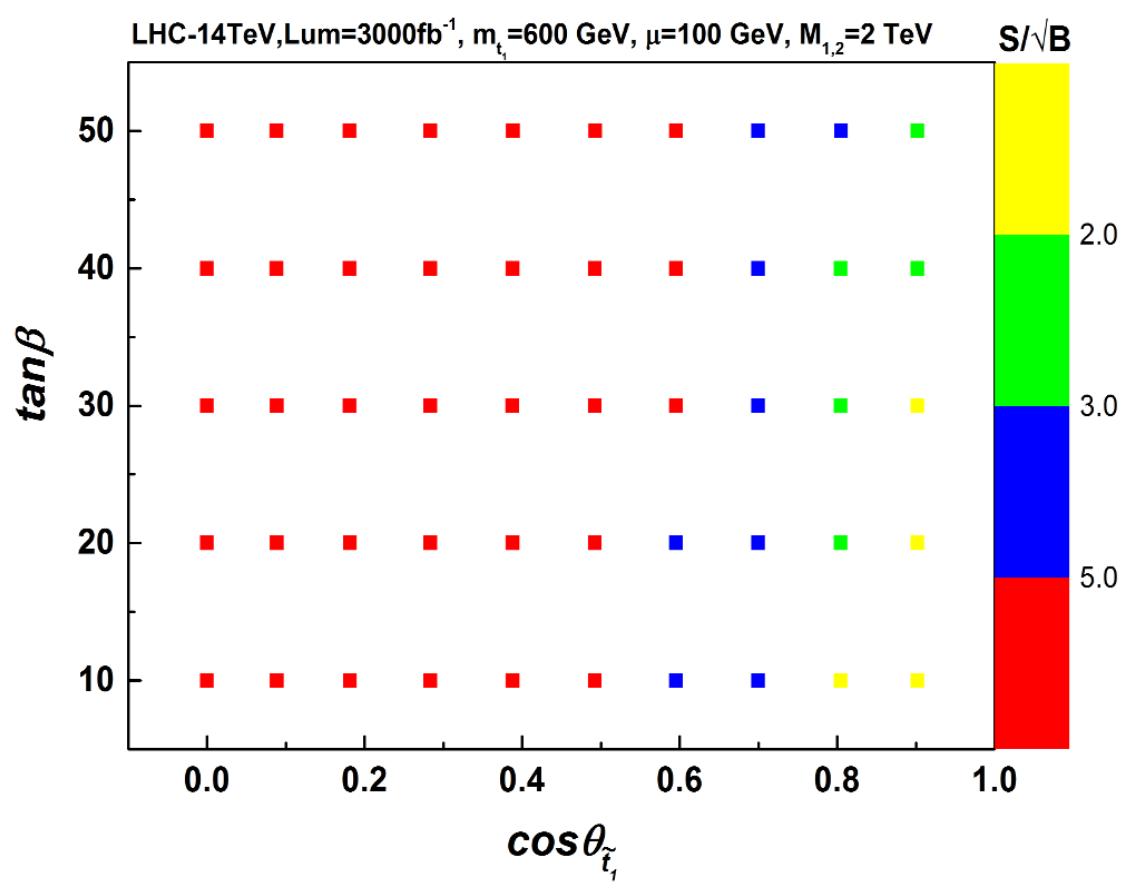

Figure 7. The statistical significance $S / \sqrt{B}$ for a benchmark point $m_{\tilde{t}_{1}}=600 \mathrm{GeV}, \mu=100 \mathrm{GeV}$ and $M_{1,2}=2 \mathrm{TeV}$ on the plane of $\cos \theta_{\tilde{t}_{1}}$ and $\tan \beta$ at $14 \mathrm{TeV}$ LHC with $\mathcal{L}=3000 \mathrm{fb}^{-1}$.

Given a discovery of the stop and a measurement of the single stop production cross section, we examine the discriminating power of single stop production with regard to the electroweak properties of the stop. In figure 7 , we show the statistical significance $S / \sqrt{B}$ of the process $p p \rightarrow \tilde{t}_{1} \tilde{\chi}_{1}^{-}$for a benchmark point $m_{\tilde{t}_{1}}=600 \mathrm{GeV}, \mu=100 \mathrm{GeV}$ and $M_{1,2}=2 \mathrm{TeV}$ on the plane of stop mixing angle $\cos \theta_{\tilde{t}_{1}}$ and $\tan \beta$ at $14 \mathrm{TeV}$ LHC with $\mathcal{L}=3000 \mathrm{fb}^{-1}$. We can see that the mixing angle $\cos \theta_{\tilde{t}_{1}} \lesssim 0.5$ ( right-handed-like stop) can be probed above $5 \sigma$ level. While for $\cos \theta_{\tilde{t}_{1}} \gtrsim 0.5$ (left-handed-like stop), the significance $S / \sqrt{B}$ depends on the value of $\tan \beta$. This is because the cross section of $\tilde{t}_{L} \tilde{H}^{-}$production is sensitive to $\tan \beta$ (cf. figure 2). When $\cos \theta_{\tilde{t}_{1}}>0.7$, the significance $S / \sqrt{B}$ is be less than $3 \sigma$. On the other hand, if $\tilde{\chi}_{1}^{-}$is wino-like, we can expect that the large $\cos \theta_{\tilde{t}_{1}}$ region will have larger $S / \sqrt{B}$ than the small $\cos \theta_{\tilde{t}_{1}}$ region at the HL-LHC since the cross section of $\tilde{t}_{L} \tilde{W}^{-}$production is much larger than that of $\tilde{t}_{R} \tilde{W}^{-}$production (cf. figure 2 ).

\section{Conclusion}

In this work we explored the observability of the associated production of stop and chargino in the compressed electroweakino scenario at $14 \mathrm{TeV}$ LHC. Due to the small mass splitting between $\tilde{\chi}_{1}^{0}$ and $\tilde{\chi}_{1}^{-}$, such a production can lead to the mono-top signature via stop decay $\tilde{t}_{1} \rightarrow t \tilde{\chi}_{1}^{0}$. We analyze the leptonic mono-top channel $p p \rightarrow \tilde{t}_{1} \tilde{\chi}_{1}^{-} \rightarrow b \ell+\mathbb{E}_{T}$, and construct a lab-frame observable $\cos \theta_{b \ell}$ from the top quark in the stop decay to reduce the SM backgrounds. We found that the stop mass can be probed up to $760 \mathrm{GeV}$ at $2 \sigma$ level through the single stop production at $14 \mathrm{TeV}$ LHC with $\mathcal{L}=3000 \mathrm{fb}^{-1}$. We also find that 
the stop mixing angle can also be determined from the single stop production assuming a measurement of the single stop production cross section at HL-LHC.

\section{Acknowledgments}

G. H. Duan thanks Yang Zhang for helpful discussions. This work is partly supported by the Australian Research Council, by the National Natural Science Foundation of China (NNSFC) under grants Nos. 11105124, 11105125, 11275057, 11305049, 11375001, 11405047, 11135003, 11275245, by the CAS Center for Excellence in Particle Physics (CCEPP) and by the CAS Key Research Program of Frontier Sciences.

Open Access. This article is distributed under the terms of the Creative Commons Attribution License (CC-BY 4.0), which permits any use, distribution and reproduction in any medium, provided the original author(s) and source are credited.

\section{References}

[1] ATLAS collaboration, Combined search for the Standard Model Higgs boson using up to $4.9 \mathrm{fb}^{-1}$ of pp collision data at $\sqrt{\mathrm{s}}=7 \mathrm{TeV}$ with the ATLAS detector at the LHC, Phys. Lett. B 710 (2012) 49 [arXiv: 1202.1408] [INSPIRE].

[2] CMS collaboration, Combined results of searches for the standard model Higgs boson in pp collisions at $\sqrt{s}=7$ TeV, Phys. Lett. B 710 (2012) 26 [arXiv:1202.1488] [InSPIRE].

[3] R. Barbieri and G.F. Giudice, Upper Bounds on Supersymmetric Particle Masses, Nucl. Phys. B 306 (1988) 63 [InSPIRE].

[4] L.J. Hall, D. Pinner and J.T. Ruderman, A Natural SUSY Higgs Near 126 GeV, JHEP 04 (2012) 131 [arXiv:1112.2703] [INSPIRE].

[5] M. Papucci, J.T. Ruderman and A. Weiler, Natural SUSY Endures, JHEP 09 (2012) 035 [arXiv: 1110.6926] [INSPIRE].

[6] C. Brust, A. Katz, S. Lawrence and R. Sundrum, SUSY, the Third Generation and the LHC, JHEP 03 (2012) 103 [arXiv:1110.6670] [INSPIRE].

[7] J. Cao, C. Han, L. Wu, J.M. Yang and Y. Zhang, Probing Natural SUSY from Stop Pair Production at the LHC, JHEP 11 (2012) 039 [arXiv: 1206.3865] [INSPIRE].

[8] Z. Kang, T. Li, J. Li and Y. Liu, A Rediatively Light Stop Saves the Best Global Fit for Higgs Boson Mass and Decays, arXiv:1208.2673 [INSPIRE].

[9] I. Low, Polarized charginos (and top quarks) in scalar top quark decays, Phys. Rev. D 88 (2013) 095018 [arXiv: 1304.0491] [INSPIRE].

[10] G.D. Kribs, A. Martin and A. Menon, Natural Supersymmetry and Implications for Higgs physics, Phys. Rev. D 88 (2013) 035025 [arXiv:1305.1313] [InSPIRE].

[11] C. Han, K.-i. Hikasa, L. Wu, J.M. Yang and Y. Zhang, Current experimental bounds on stop mass in natural SUSY, JHEP 10 (2013) 216 [arXiv:1308.5307] [INSPIRE].

[12] K. Kowalska and E.M. Sessolo, Natural MSSM after the LHC 8 TeV run, Phys. Rev. D 88 (2013) 075001 [arXiv:1307.5790] [INSPIRE]. 
[13] M. Backović, A. Mariotti and M. Spannowsky, Signs of Tops from Highly Mixed Stops, JHEP 06 (2015) 122 [arXiv: 1504.00927] [INSPIRE].

[14] A. Kobakhidze, N. Liu, L. Wu and J.M. Yang, ATLAS Z-peaked excess in the MSSM with a light sbottom or stop, Phys. Rev. D 92 (2015) 075008 [arXiv:1504.04390] [INSPIRE].

[15] B. Dutta et al., Probing compressed top squark scenarios at the LHC at $14 \mathrm{TeV}$, Phys. Rev. D 90 (2014) 095022 [arXiv: 1312.1348] [INSPIRE].

[16] G. Bélanger, D. Ghosh, R. Godbole and S. Kulkarni, Light stop in the MSSM after LHC Run 1, JHEP 09 (2015) 214 [arXiv:1506.00665] [INSPIRE].

[17] A. Kobakhidze, N. Liu, L. Wu, J.M. Yang and M. Zhang, Closing up a light stop window in natural SUSY at LHC, Phys. Lett. B 755 (2016) 76 [arXiv:1511.02371] [InSPIRE].

[18] M. Drees and J.S. Kim, Minimal natural supersymmetry after the LHC8, Phys. Rev. D 93 (2016) 095005 [arXiv: 1511.04461] [INSPIRE].

[19] H. Baer, V. Barger, M. Savoy and X. Tata, Multichannel assault on natural supersymmetry at the high luminosity LHC, Phys. Rev. D 94 (2016) 035025 [arXiv:1604.07438] [inSPIRE].

[20] J. Fan, R. Krall, D. Pinner, M. Reece and J.T. Ruderman, Stealth Supersymmetry Simplified, JHEP 07 (2016) 016 [arXiv:1512.05781] [INSPIRE].

[21] M. Schlaffer, M. Spannowsky and A. Weiler, Searching for supersymmetry scalelessly, Eur. Phys. J. C 76 (2016) 457 [arXiv: 1603.01638] [INSPIRE].

[22] D. Gonçalves, K. Sakurai and M. Takeuchi, Mono-top Signature from Supersymmetric t $\bar{t} H$ Channel, Phys. Rev. D 94 (2016) 075009 [arXiv: 1604.03938] [INSPIRE].

[23] D. Goncalves, K. Sakurai and M. Takeuchi, Tagging a monotop signature in natural SUSY, Phys. Rev. D 95 (2017) 015030 [arXiv:1610.06179] [INSPIRE].

[24] C.G. Lester and D.J. Summers, Measuring masses of semiinvisibly decaying particles pair produced at hadron colliders, Phys. Lett. B 463 (1999) 99 [hep-ph/9906349] [INSPIRE].

[25] A. Barr, C. Lester and P. Stephens, A variable for measuring masses at hadron colliders when missing energy is expected; $m_{T 2}$ : the truth behind the glamour, J. Phys. G 29 (2003) 2343 [hep-ph/0304226] [INSPIRE].

[26] M. Drees, M. Hanussek and J.S. Kim, Light Stop Searches at the LHC with Monojet Events, Phys. Rev. D 86 (2012) 035024 [arXiv:1201.5714] [inSPIRE].

[27] S. Bornhauser, M. Drees, S. Grab and J.S. Kim, Light Stop Searches at the LHC in Events with two b-Jets and Missing Energy, Phys. Rev. D 83 (2011) 035008 [arXiv:1011.5508] [INSPIRE].

[28] Z.-H. Yu, X.-J. Bi, Q.-S. Yan and P.-F. Yin, Detecting light stop pairs in coannihilation scenarios at the LHC, Phys. Rev. D 87 (2013) 055007 [arXiv:1211.2997] [INSPIRE].

[29] M.A. Ajaib, T. Li and Q. Shafi, Stop-Neutralino Coannihilation in the Light of LHC, Phys. Rev. D 85 (2012) 055021 [arXiv: 1111.4467] [INSPIRE].

[30] K. Hagiwara and T. Yamada, Equal-velocity scenario for hiding dark matter at the LHC, Phys. Rev. D 91 (2015) 094007 [arXiv: 1307.1553] [INSPIRE].

[31] H. An and L.-T. Wang, Opening up the compressed region of top squark searches at $13 \mathrm{TeV}$ LHC, Phys. Rev. Lett. 115 (2015) 181602 [arXiv:1506. 00653] [INSPIRE]. 
[32] S. Macaluso, M. Park, D. Shih and B. Tweedie, Revealing Compressed Stops Using High-Momentum Recoils, JHEP 03 (2016) 151 [arXiv: 1506. 07885] [INSPIRE].

[33] ATLAS collaboration, Search for new phenomena in final states with an energetic jet and large missing transverse momentum in pp collisions at $\sqrt{s}=13$ TeV using the ATLAS detector, Phys. Rev. D 94 (2016) 032005 [arXiv: 1604.07773] [INSPIRE].

[34] CMS collaboration, An inclusive search for new phenomena in final states with one or more jets and missing transverse momentum at $13 \mathrm{TeV}$ with the AlphaT variable, CMS-PAS-SUS-16-016 (2016).

[35] C. Han, J. Ren, L. Wu, J.M. Yang and M. Zhang, Top-squark in natural SUSY under current LHC run-2 data, Eur. Phys. J. C 77 (2017) 93 [arXiv:1609.02361] [INSPIRE].

[36] J.S. Kim, K. Rolbiecki, R. Ruiz, J. Tattersall and T. Weber, Prospects for natural SUSY, Phys. Rev. D 94 (2016) 095013 [arXiv: 1606. 06738] [INSPIRE].

[37] K. Kowalska, Phenomenological MSSM in light of new 13 TeV LHC data, Eur. Phys. J. C 76 (2016) 684 [arXiv : 1608.02489] [INSPIRE].

[38] C. Han, M.M. Nojiri, M. Takeuchi and T.T. Yanagida, Surviving scenario of stop decays for $A T L A S \ell+j e t s+E_{T}^{\text {miss }}$ search, Phys. Lett. B 767 (2017) 37 [arXiv:1609.09303] [INSPIRE].

[39] K. Kowalska and E.M. Sessolo, MSSM fits to the ATLAS 1 lepton excess, Eur. Phys. J. C 77 (2017) 79 [arXiv:1611.01852] [INSPIRE].

[40] A. Barr and J. Liu, First interpretation of 13 TeV supersymmetry searches in the pMSSM, arXiv: 1605.09502 [INSPIRE].

[41] D. Goncalves, D. Lopez-Val, K. Mawatari and T. Plehn, Automated third generation squark production to next-to-leading order, Phys. Rev. D 90 (2014) 075007 [arXiv:1407.4302] [INSPIRE].

[42] A. Ismail, R. Schwienhorst, J.S. Virzi and D.G.E. Walker, Deconstructed Transverse Mass Variables, Phys. Rev. D 91 (2015) 074002 [arXiv:1409.2868] [INSPIRE].

[43] K.-i. Hikasa, J. Li, L. Wu and J.M. Yang, Single top squark production as a probe of natural supersymmetry at the LHC, Phys. Rev. D 93 (2016) 035003 [arXiv:1505.06006] [INSPIRE].

[44] N. Arkani-Hamed, A. Delgado and G.F. Giudice, The well-tempered neutralino, Nucl. Phys. B 741 (2006) 108 [hep-ph/0601041] [INSPIRE].

[45] J. Andrea, B. Fuks and F. Maltoni, Monotops at the LHC, Phys. Rev. D 84 (2011) 074025 [arXiv:1106.6199] [INSPIRE].

[46] J. Wang, C.S. Li, D.Y. Shao and H. Zhang, Search for the signal of monotop production at the early LHC, Phys. Rev. D 86 (2012) 034008 [arXiv:1109.5963] [INSPIRE].

[47] J.-L. Agram, J. Andrea, M. Buttignol, E. Conte and B. Fuks, Monotop phenomenology at the Large Hadron Collider, Phys. Rev. D 89 (2014) 014028 [arXiv: 1311.6478] [InSPIRE].

[48] E. Alvarez, E. Coluccio Leskow, J. Drobnak and J.F. Kamenik, Leptonic Monotops at LHC, Phys. Rev. D 89 (2014) 014016 [arXiv:1310.7600] [inSPIRE].

[49] I. Boucheneb, G. Cacciapaglia, A. Deandrea and B. Fuks, Revisiting monotop production at the LHC, JHEP 01 (2015) 017 [arXiv: 1407.7529] [INSPIRE].

[50] J.F. Gunion and H.E. Haber, Higgs Bosons in Supersymmetric Models. 1., Nucl. Phys. B 272 (1986) 1 [Erratum ibid. B 402 (1993) 567] [INSPIRE]. 
[51] G.F. Giudice and A. Pomarol, Mass degeneracy of the Higgsinos, Phys. Lett. B 372 (1996) 253 [hep-ph/9512337] [INSPIRE].

[52] C. Han, A. Kobakhidze, N. Liu, A. Saavedra, L. Wu and J.M. Yang, Probing Light Higgsinos in Natural SUSY from Monojet Signals at the LHC, JHEP 02 (2014) 049 [arXiv: 1310.4274] [INSPIRE].

[53] Z. Han, G.D. Kribs, A. Martin and A. Menon, Hunting quasidegenerate Higgsinos, Phys. Rev. D 89 (2014) 075007 [arXiv:1401.1235] [INSPIRE].

[54] C. Han, D. Kim, S. Munir and M. Park, Accessing the core of naturalness, nearly degenerate higgsinos, at the LHC, JHEP 04 (2015) 132 [arXiv: 1502.03734] [INSPIRE].

[55] D. Barducci, A. Belyaev, A.K.M. Bharucha, W. Porod and V. Sanz, Uncovering Natural Supersymmetry via the interplay between the LHC and Direct Dark Matter Detection, JHEP 07 (2015) 066 [arXiv: 1504.02472] [INSPIRE].

[56] J. Bramante, A. Delgado, F. Elahi, A. Martin and B. Ostdiek, Catching sparks from well-forged neutralinos, Phys. Rev. D 90 (2014) 095008 [arXiv: 1408.6530] [InSPIRE].

[57] C. Han, L. Wu, J.M. Yang, M. Zhang and Y. Zhang, New approach for detecting a compressed bino/wino at the LHC, Phys. Rev. D 91 (2015) 055030 [arXiv:1409.4533] [INSPIRE].

[58] A. Ismail, E. Izaguirre and B. Shuve, Illuminating New Electroweak States at Hadron Colliders, Phys. Rev. D 94 (2016) 015001 [arXiv: 1605.00658] [INSPIRE].

[59] A. Djouadi, J.-L. Kneur and G. Moultaka, SuSpect: A Fortran code for the supersymmetric and Higgs particle spectrum in the MSSM, Comput. Phys. Commun. 176 (2007) 426 [hep-ph/0211331] [INSPIRE].

[60] A. Djouadi, M.M. Muhlleitner and M. Spira, Decays of supersymmetric particles: The Program SUSY-HIT (SUspect-SdecaY-HDECAY-InTerface), Acta Phys. Polon. B 38 (2007) 635 [hep-ph/0609292] [INSPIRE].

[61] J. Alwall et al., The automated computation of tree-level and next-to-leading order differential cross sections and their matching to parton shower simulations, JHEP 07 (2014) 079 [arXiv: 1405.0301] [INSPIRE].

[62] NNPDF collaboration, R.D. Ball et al., Parton distributions with QED corrections, Nucl. Phys. B 877 (2013) 290 [arXiv: 1308.0598] [INSPIRE].

[63] T. Sjöstrand, S. Mrenna and P.Z. Skands, PYTHIA 6.4 Physics and Manual, JHEP 05 (2006) 026 [hep-ph/0603175] [INSPIRE].

[64] DELPHES 3 collaboration, J. de Favereau et al., DELPHES 3, A modular framework for fast simulation of a generic collider experiment, JHEP 02 (2014) 057 [arXiv:1307.6346] [INSPIRE].

[65] M. Cacciari, G.P. Salam and G. Soyez, The anti-k $k_{t}$ jet clustering algorithm, JHEP 04 (2008) 063 [arXiv: 0802.1189] [INSPIRE].

[66] N. Kidonakis, The top quark rapidity distribution and forward-backward asymmetry, Phys. Rev. D 84 (2011) 011504 [arXiv: 1105.5167] [INSPIRE].

[67] Particle Data Group collaboration, C. Patrignani et al., Review of Particle Physics, Chin. Phys. C 40 (2016) 100001 [InSPIRE]. 